\title{
The J-lndex as a Measure of Nominal Scale Response Agreement
}

\author{
Svante Janson and Jan Vegellus \\ Universiey of Uppala
}

\begin{abstract}
Some characteristics of Hubert's $\Gamma$, as a measure of nominal scale response agreement, are shown, including the characteristic that in a contingency table with equal frequencies its value will normally not be zero. By making a slight modification of its definition, some of these characteristics can be
\end{abstract}

\begin{abstract}
eliminated. As another alternative, the $J$-index is suggested. It is closely related to $r$ but does not have the same problematic characteristics. Some asymptotic variance formulas for the $\mathbb{J}$-index are given, together with a numerical example.
\end{abstract}

Measuring response agreements between two judges by measures of association seems to be a natural choice. For situations in which both judges use the same nominal scale for their judgments, $\mathrm{Co}$ hen's kappa (weighted or not; Cohen, 1960, 1968) is a possible alternative. Two other suitable coefficients for this case are the $S$ - and $C$-indices (Janson \& Vegelius, 1979). If, however, the two judges apply different nominal scales, the coefficients mentioned are not possible to use. For this case Hubert (1977) suggested an interesting alternative, based on some considerations by Brennan and Light (1974).

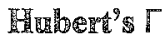

Suppose two raters, $\mathrm{A}$ and $\mathrm{B}$, assign $n$ objects in a set $S$ to a number of nominal classes. Assume that rater $\mathrm{A}$ uses $R$ (nonempty) classes $\left\{A_{1}, A_{2}, \ldots, A_{R}\right\}$ and rater $\mathrm{B}$ uses $C$ (nonempty) classes $\left\{B_{1}, B_{2}, \ldots, \mathbb{B}_{C}\right\}$. Let $n_{i j}$ denote the number of objects placed in category $A_{i}$ by judge $A$ and placed in category $B_{j}$ by judge $B$. Thus, an $R \times C$ contingency table is obtained where standard notations are used for the various marginal sums (e.g., Table 1 ).

Suppose the data are presented in the form of a sequence of $n$ bivariate (non-numerical) observations:

$\left(\mathbb{A}, \mathbb{B}_{1}\right), \ldots,\left(\mathbb{A}_{n}, \mathbb{B}_{n}\right)$

where $\left(\mathbb{A}_{k}, \mathbb{B}_{k}\right)=\left(A_{i}, B_{j}\right)$ whenever rater 1 places the $k^{\text {th }}$ object from $S$ into category $A_{i}$ and rater 2 places the same object into category $B_{j}$. Furthermore, let $\mathbb{A}=\left\{\mathbb{A}_{1}, \mathbb{A}_{2}, \ldots, \mathbb{A}_{n}\right\}, \mathbb{B}=\left\{\mathbb{B}_{1}, \mathbb{B}_{2}, \ldots, \mathbb{R}_{n}\right\}$ and

APPLIED PSYCHOLOGICAL MEASUREMENT

Vol. 6, No. 1, Winter 1982, pp. 111-121

(C) Copyright 1982 Applied Psychological Measurement Inc.

$0146-6216 / 82 / 010111-11 \$ 1.55$ 
Table 1

Contingency Table of Number of Assignments
by Two Raters

\begin{tabular}{|c|c|c|c|c|c|}
\hline \multirow[b]{2}{*}{ Rater 1} & \multicolumn{5}{|c|}{ Rater 2} \\
\hline & $\mathrm{B}_{1}$ & $\mathrm{~B}_{2}$ & $\cdots$ & ${ }^{B} \mathrm{C}$ & Sums \\
\hline $\mathrm{A}_{1}$ & $\mathrm{n}_{11}$ & $\mathrm{n}_{12}$ & $\ldots \ldots$ & $\mathrm{n}_{1 \mathrm{C}}$ & $\mathrm{n}_{1}$. \\
\hline$A_{2}$ & $\mathrm{n}_{21}$ & $n_{22}$ & $\ldots \ldots$ & ${ }^{\mathrm{n}} 2 \mathrm{C}$ & $\mathrm{n}_{2}$ \\
\hline • & 。 & 。 & & 。 & 。 \\
\hline 。 & . & - & & - & - \\
\hline 。 & 。 & 。 & & . & 。 \\
\hline $\mathrm{A}_{\mathrm{R}}$ & $\mathrm{n}_{\mathrm{R} 1}$ & ${ }^{n}{ }_{R}$ & $\ldots \ldots$ & ${ }^{\mathrm{n}_{\mathrm{RC}}}$ & $\mathrm{n}_{\mathbb{R}}$. \\
\hline Sums & $n \cdot 1$ & $\mathrm{n} .2$ & $\ldots \ldots$ & $\mathrm{n}, \mathrm{C}$ & $\mathrm{n}_{0} \mathrm{a}=\mathrm{n}$ \\
\hline
\end{tabular}

define the following two functions on $\mathbb{A} \times \mathbb{A}$ and $\mathbb{B}$ corresponding to rater 1 and rater 2 , respectively:

$$
Q\left(\mathbb{A}_{S}, \mathbb{A}_{t}\right)=\left\{\begin{array}{l}
1 \text { if } \mathbb{A}_{S}=\mathbb{A}_{t} \\
-1 \text { if } \mathbb{A}_{S} \neq \mathbb{A}_{t}
\end{array} \quad Q\left(\mathbb{B}_{S}, \mathbb{B}_{t}\right)=\left\{\begin{array}{l}
1 \text { if } \mathbb{B}_{S}=\mathbb{B}_{t} \\
-1 \text { if } \mathbb{B}_{S} \neq \mathbb{B}_{t}
\end{array}\right.\right.
$$

for $1 \leqslant s \neq t \leqslant n$; also, it is assumed as a technical convenience that $Q\left(\mathbb{A}_{s}, \mathbb{A}_{s}\right)=Q\left(\mathbb{B}_{s}, \mathbb{B}_{s}\right)=0$ for $1 \leqslant s \leqslant n$. Given these preliminaries, Daniel's concept of a generalized correlation coefficient (see Kendall, 1970) can be extended by analogy to include a natural index of response agreement defined formally by

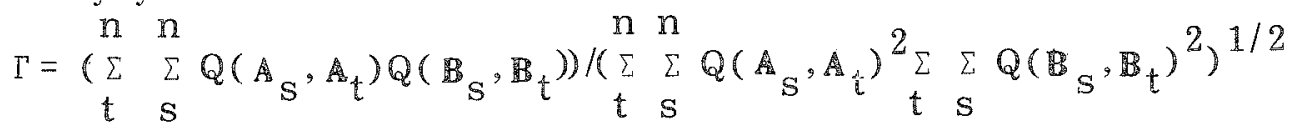

It is easily shown that

$$
\begin{aligned}
\Gamma & =(A-D) /(A+D)=(A-D) /\left(\begin{array}{l}
n \\
2
\end{array}\right)= \\
& =1+\left[\begin{array}{lllll}
C & R & R \\
2 & \sum_{j} & \sum_{i} & n_{i j}^{2} \\
j & -\left(\sum_{i}\right. & n_{i}^{2}+\sum_{j}^{2} & n^{2}{ }^{2} j
\end{array}\right] /\left(\begin{array}{l}
n \\
2
\end{array}\right) ;
\end{aligned}
$$

where

$$
A=\left(\begin{array}{l}
n \\
2
\end{array}\right)+\sum_{i}^{R} \sum_{j} n_{i j}^{2}-\frac{1}{2}\left(\sum_{i}^{R} n_{i \circ}^{2}+\sum_{j}^{C} n_{\circ j}^{2}\right)
$$

and

$$
\mathbb{D}=\left(\begin{array}{l}
n \\
2
\end{array}\right)-A
$$


Hubert's $\Gamma$ has many appealing characteristics. As it is a special case of Daniel-Kendall's generalized correlation coefficient, it is also an $E$-correlation coefficient (Vegelius, 1978a), i.e., it satisfies the requirements of a scalar product between normalized vectors in a Euclidean space. This implies that, for example, a components analysis may be based on $r$. However, there are some characteristics of Hubert's $\Gamma$ that might be considered less suitable for such a coefficient.

Let all $n_{i j}$ be equal. Then, Equation 4 will become

$$
\Gamma=\left(\frac{n}{n-1}\right) \cdot\left(1-\frac{2}{\mathrm{C}}\right) \cdot\left(1-\frac{2}{\mathrm{R}}\right)-\frac{1}{(n-1)}
$$

From Equation 7 the following two characteristics of $\Gamma$ are deduced.

Characteristic 1: If $C \geqslant 3$ and $R \geqslant 3$, Equation 7 shows that $\Gamma$ is positive when all frequencies are equal (with one exception, namely, when $R=C=3$ and all $n_{i j}=1$ ). A reasonable requirement for a measure of response agreement is that it should equal zero when all frequencies are equal. $\Gamma$ does not satisfy that requirement.

Characteristic 2: If, instead, $C=2$ or $R=2$, Equation 7 shows that $\Gamma=-[1 /(n-1)]$, although all cell frequencies are equal. As above, the value zero would be preferable.

Now consider an arbitrary $2 \times 2$ table. For such a table is deduced

$$
\Gamma=1-4 \cdot \frac{\left(n_{11}+n_{22}\right)\left(n_{12}+n_{21}\right)}{n \cdot(n-1)}
$$

From Equation 8 it follows that

$$
\Gamma \geq-\frac{1}{(n-1)}
$$

where equality holds if and only if

$$
\left(n_{11}+n_{22}\right)=\frac{n}{2}
$$

From these equations two other characteristics of $\Gamma$ may be deduced.

Characteristic 3: $\Gamma$ may thus be negative, having $-[1 /(n-1)]$ as its minimum value. It may seem odd for a measure of response agreement between persons using different nominal scale variables to have negative values. It may be considered as more reasonable with zero as the minimum value.

Characteristic 4: Equation 8 shows how $\Gamma$ works for dichotomized data. This equation does not seem to be closely related to other association measures between dichotomized variables.

Now it is rather easy to eliminate two of these characteristics by modifying $\Gamma$ somewhat. This is done by eliminating the extra definition of $Q\left(\mathbb{A}_{s}, \mathbb{A}_{s}\right)$ and $Q\left(\mathbb{B}_{s}, \mathbb{B}_{s}\right)$.

Thus,

$$
Q\left(\mathbb{A}_{S}, A_{S}\right)=Q\left(\mathbb{B}_{S}, \mathbb{R}_{S}\right)=1 \quad 1 \leq \mathrm{s} \leq \mathrm{n}
$$

This modified coefficient will be called $\Gamma *$. Its general formula is

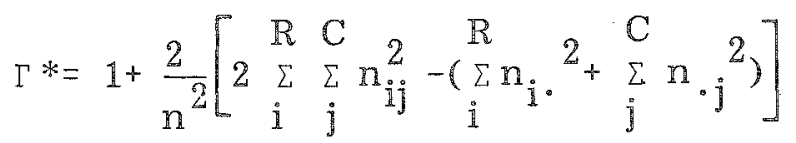


If all $n_{i j}$ are equal, it follows that

$$
\Gamma *\left(1-\frac{2}{\mathrm{C}}\right) \cdot\left(1-\frac{2}{\mathrm{R}}\right)
$$

From Equation 13 it follows that $\Gamma$ will be positive if $C \geqslant 3$ and $k \geqslant 3$, i.e., if Characteristic 1 still applies. On the other hand, Characteristic 2 is now eliminated. For a general $2 \times 2$ table is deduced:

$$
\Gamma *=1-4 \cdot \frac{\left(n_{11}+n_{22}\right) \cdot\left(n_{12}+n_{21}\right)}{n^{2}}
$$

From Equation 14 it follows that Characteristic 3 is also eliminated but not Characteristic 4. Characteristics 1 and 4 are more fundamental. The next section will describe an alternative to $\Gamma$ (and $\Gamma *)$, which is related to $\Gamma$ but does not have any of these four characteristics.

\section{The Julpor}

Recently a new correlation coefficient for nominal scales was introduced under the designation the $J$-index (Janson \& Vegelius, 1978). The $J$-index is also possible to use as a measure of response agreement when the two raters use different nominal scale judgements. The $J$-index is defined as the special case of Kendall's general coefficient (Equation 2), where

$$
Q\left(\mathbb{A}_{\mathrm{S}}, \mathbb{A}_{t}\right)=\left(\begin{array}{c}
\mathbb{R}-1 \text { if } A_{S}=A_{t} \\
-1 \text { if } A_{S} \neq \mathbb{A}_{t} \\
Q\left(\mathbb{B}_{S}, \mathbb{B}_{t}\right)= \\
-1 \text { if } \mathbb{B}_{S} \neq \mathbb{R}_{t}
\end{array}\right.
$$

(There is no special definition of $Q\left(\mathbb{A}_{s}, A_{s}\right)$ or $Q\left(\mathbb{R}_{s}, \mathbb{R}_{s}\right)$ )

It may be shown (Janson \& Vegelius, 1978) that if $C \geqslant 2$ and $R \geqslant 2, J$ can be developed into

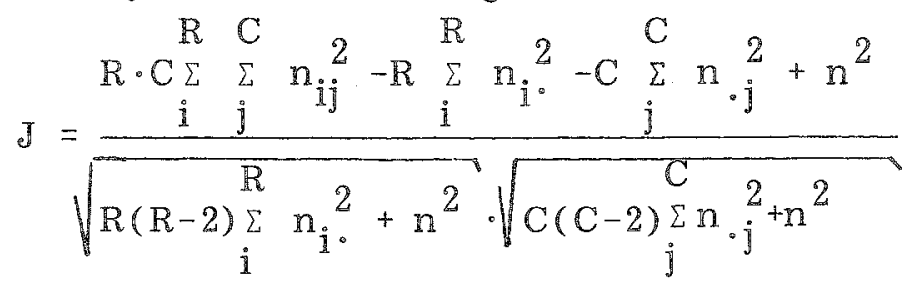

$$
=\frac{R \cdot C \cdot \sum_{i}^{R} \sum_{j}^{C} p_{i j}^{2}-R \sum_{i}^{R} p_{i}^{2}-C \sum_{j} p_{\cdot j}^{2}+1}{\sqrt{R(R-2) \cdot \sum_{i}^{R} p_{i}^{2}+1 \cdot \sqrt{C(C-2) \sum_{j}^{2} p_{\circ j}^{2}+1}}}
$$


where

$$
\begin{aligned}
& p_{i j}=n_{i j} / n \\
& p_{i}=n_{i} / n \\
& p_{. j}=n_{. j} / n
\end{aligned}
$$

It is easily verified (cf. Janson \& Vegelius, 1978) that when all cell frequencies are equal, $J$ will equal 0 , i.e., Characteristics 1 and 2 do not affect the $J$-index.

For dichotomized data

$$
J=\frac{\left[\left(n_{11}+n_{22}\right)-\left(n_{12}+n_{21}\right)\right]^{2}}{n^{2}}
$$

i.e., it is equal to the square of the $G$-index (Holley \& Gullord, 1964). From this it follows that Characteristics 3 and 4 do not affect the $J$-index either. Thus, the $J$-index is an alternative to Hubert's $\Gamma$, closely related to it but without the four characteristics discussed.

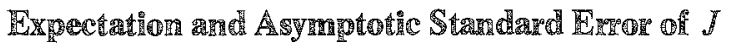

The population parameter corresponding to $J$ will here be denoted by $\mathbb{I}$. If $\pi_{i j}$ denotes the probability that judge $A$ assigns category $\mathbb{A}_{i}$ and judge $B$ category $B_{j}$ to a randomly chosen unit, thent $\mid$ will be defined as

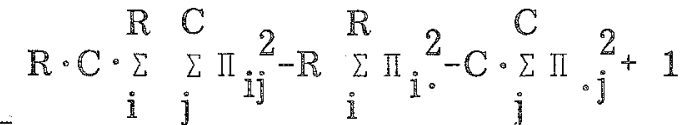

$$
\begin{aligned}
& 1 \\
& \sqrt{R(R-2) \sum_{i}^{R} \Pi_{i}^{2}+1 \sqrt{C(C-2) \cdot \sum_{j} \pi_{0}^{2}+1}}
\end{aligned}
$$

The following symbols will be used:

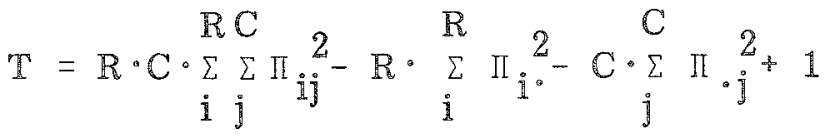

$$
\begin{aligned}
& S_{R}=\sqrt{R \cdot(R-2) \sum_{i} \pi_{1}^{2}+1} \\
& S_{C}=\sqrt{C(C-2) \cdot \sum_{j} \pi_{i j}^{2}+1}
\end{aligned}
$$

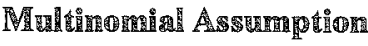

Assume that the $R \times C$ contingency table is generated rom a single multinomial distribution with parameters $\left\{\pi_{i j}\right\}$, where $\sum_{i=1}^{R} \sum_{j=1}^{C} \pi_{i j}=1$. The number of degrees of freedom is thus $R \cdot C-1$. According to Rao (1965; cf. Cramér, 1945), the following equation for the asymptotic variance can be utilized:

$$
\mathrm{D}^{2}(J)=\frac{1}{n}\left[\frac{\partial J}{\partial \mathrm{p}}\right]_{\mathrm{p}=\pi} \cdot \Sigma_{\mathrm{p}} \cdot\left[\frac{\partial \mathrm{J}}{\partial \mathrm{p}}\right]_{\mathrm{p}=\pi}
$$


where $[\partial J / \partial p]_{p=r}$ is a column matrix consisting of the derivatives of $J$ with respect to the proportions of the $R \times C$ cells and where $n^{-1} \cdot \Sigma p$ is the covariance matrix of the observed proportions. The elements in $\Sigma p$ are

$$
\sigma_{i j, k l}=\left\{\begin{array}{l}
\pi_{i j} \cdot\left(1-\pi_{i j}\right) \text { if }(i, j)=(k, 1) \\
-\pi_{i j} \cdot \pi_{k 1} \text { if }(i, j) \neq(k, 1)
\end{array}\right.
$$

The elements of $[\partial J / \partial p]_{p=\pi}$ are

$$
\left[\frac{\partial J}{\partial p_{i j}}\right]_{p_{i j}=\pi_{i j}}=\frac{D \cdot T_{i j}^{\prime}-D_{i j}^{\prime} \cdot T}{D^{2}}
$$

where $\mathbb{D}=S_{R} \cdot S_{C}$

$$
\begin{aligned}
& D_{i j}^{i}=\frac{S_{R}}{S_{C}} \cdot C(C-2) \cdot \pi_{\cdot j}+\frac{S_{C}}{S_{R}} \cdot R(R-2) \cdot \pi_{i} \\
& T_{i j}^{i}=2 \cdot R \cdot C \cdot \pi_{i j}-2 \cdot R \cdot \pi_{i}-2 \cdot C \cdot \pi_{a j}
\end{aligned}
$$

For simplicity reasons the denotation $\left[\partial J / \partial \pi_{i j}\right]$ will be used instead of $\left[\partial J / \partial p_{i j}\right]_{p_{i j}=\pi_{i j}}$. Equation 22 is now equivalent to

$$
\begin{aligned}
& n \cdot D^{2}(J)=\begin{array}{cccc}
R & C & R & C \\
i & \sum & \sum & \sum \\
i & j & k & 1
\end{array}\left(-\pi_{i j} \cdot \pi_{k l} \cdot \frac{\partial J}{\partial \pi_{i j}} \cdot \frac{\partial J}{\partial \pi_{k l}}\right)
\end{aligned}
$$

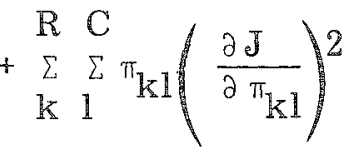

or still more simplified

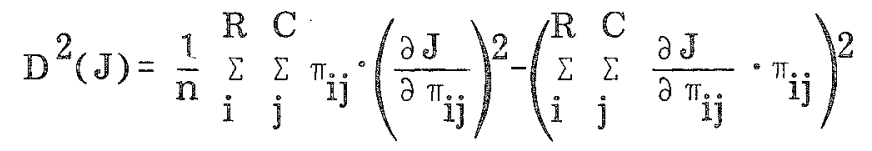

This equation may be used to obtain the asymptotic variance unless the factor of $1 / n$ equals 0 . It may be shown that this happens if and only if $I$ is 0 . By using the analogue of Equation 22 with second derivatives for the case $\mathrm{I}=0$, the following two equations may be deduced:

$$
E(J)=\frac{(R-1)(C-1)}{n \cdot S_{R} S_{C}}
$$

and 


$$
D^{2}(J)=\frac{1}{n^{2}}\left[\frac{2(C-1)}{S_{C}^{2}}+\frac{2(R-1)}{S_{R}^{2}}-\frac{2(R-1)(C-1)}{S_{C}^{2} \cdot S_{R}^{2}}\right]
$$

It is obvious that the larger $I$ is, the more satisfactory is Equation 28 for practical purposes. When used empirically, the observed proportions $p_{i j}$ are used to estimate the probabilities $\pi_{i j}$ in the equations above.

Here an interesting comparison with the determination coefficient $r^{2}$ may be made (cf. Cramér, 1945, p. 415). When $\varrho^{2} \neq 0$, the asymptotic variance of $r^{2}$ is of order $n^{-1}$; but when $\varrho^{2}=0$, the variance of $r^{2}$ is of order $n^{-2}$, just like the $J$-index.

\section{Fixed Mariginal Assumption}

In some selection situations it may be natural to consider the marginals fixed. Each judge may then decide beforehand how many he or she will assign to each of the groups.

It is here assumed that all marginal frequencies are fixed. This means that the degrees of freedom are $(R-1) \times(C-1)$. The marginal proportions are also fixed, of course.

In this section some comparisons with Hubert's $\Gamma$ are made. Therefore, the development here has many similarities with the corresponding section in his article.

The following symbol will be used here:

$\Lambda=2 \cdot(\mathrm{A}-\mathrm{D})$

$T, S_{R}$, and $S_{C}$ from Equations 19 through 21 are now to be used but with proportions instead of probabilities in the expression. A comparison between Equation 4 and Equation 19 implies

$$
T=R \cdot C \cdot \frac{(n-1)}{4 n} \cdot I+R \cdot \frac{(C-2)}{2} \cdot \sum_{i}^{R} p_{i}^{2}+C \frac{(R-2)}{2} \cdot \sum_{j}^{C} p_{\cdot j}^{2}+\left(1-\frac{R C}{4}\right)+\frac{R C}{4 n}
$$

It is also know that

$$
\Lambda=\Gamma \cdot n \cdot(n-1)
$$

As the marginal proportions are fixed,

$$
E(T)=\frac{R C}{4} \cdot \frac{E(\Lambda)}{n^{2}}+R \frac{(C-2)}{2} \cdot \sum_{i}^{R} p_{i}^{2}+C \cdot \frac{(R-2)}{2} \cdot \sum_{j}^{C} p_{j}^{2}+\left(1-\frac{R C}{4}\right)+\frac{R C}{4 n}
$$

According to Hubert (1977), the following equality holds:

$$
E(\Lambda)=\frac{1}{n \cdot(n-1)} \cdot A_{1} \cdot B_{1}
$$

where

$$
\begin{aligned}
& A_{1}=2 \cdot \sum_{i}^{R} n_{i}^{2}-(n+1) \cdot n \\
& B_{1}=2 \cdot \sum_{j} n_{0 j}^{2}-(n+1) \cdot n
\end{aligned}
$$

From this is deduced 


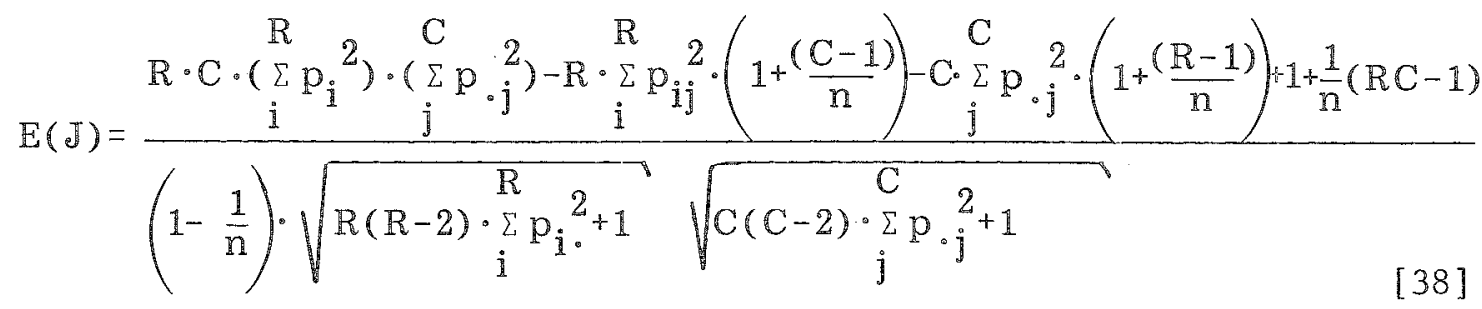

If now $n$ grows to infinity, $E(J)$ will approach

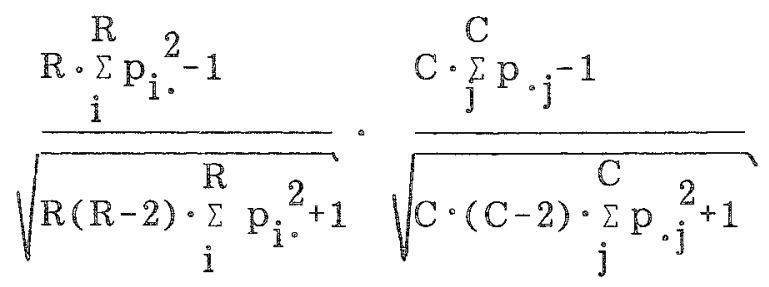

As the marginal distributions are considered to be fixed, the asymptotic variance will be

$$
D^{2}(J)=\frac{R^{2} \cdot C^{2} \cdot D^{2}(\Lambda)}{16 \cdot n^{4} \cdot S_{R}^{2} \cdot S_{C}^{2}}
$$

According to Hubert (1977), it can be stated

$$
\begin{aligned}
D^{2}(n)= & 2 n(n-1)-\left\{1 /(n(n-1)) A_{1} B_{1}\right\}^{2}+(4 /(n(n-1)(n-2)))\left(A_{2}-A_{3}\right)\left(B_{2}-B_{3}\right) \\
& +(1 /(n(n-1)(n-2)(n-3)))\left(A_{1}^{2}-4 A_{2}+2 A_{3}\right)\left(B_{1}^{2}-4 B_{2}+2 B_{3}\right)
\end{aligned}
$$

where

$$
\begin{aligned}
& A_{2}=\underset{i}{4 \sum n_{i}}{ }^{3}-4(n+1) \sum_{i}^{R} n_{i}^{2}+(n+1)^{2} n \\
& B_{2}=\underset{j}{4 \sum n} \cdot j^{3}-4(n+1) \underset{j}{C} n_{\cdot j}^{2}+(n+1)^{2} n \\
& \mathrm{~A}_{3}=\mathbb{B}_{3}=\mathrm{n}(\mathrm{n}-1)
\end{aligned}
$$

According to Hubert (1977), a large sample approximation is obtained as

$$
\mathrm{D}^{2}(\Gamma)=(4 / \mathrm{n})\left(\overline{\mathrm{A}}_{1}^{2}-\overline{\mathrm{A}}_{2}\right)\left(\overline{\mathrm{B}}_{1}{ }^{2}-\overline{\mathrm{B}}_{2}\right)
$$

where

$$
\begin{aligned}
& \bar{A}_{1}=\underset{\substack{\mathbb{1} \\
2}}{\mathbb{R}}\left(n_{i} / n\right)^{2}-1 \\
& \bar{B}_{1}=\underset{j}{2} \underset{j}{2}\left(n_{j} / n\right)^{2}-1
\end{aligned}
$$




$$
\begin{aligned}
& \bar{A}_{2}=\underset{i}{R}\left(n_{i} \cdot / n\right)^{3} \underset{-4}{R} \underset{i}{R}\left(n_{i} \cdot / n\right)^{2}+1 \\
& \bar{B}_{2}=\underset{j}{\mathbb{4}} \underset{j}{C}(n \cdot j / n)^{3}-\underset{j}{C}(n \cdot j / n)^{2}+1
\end{aligned}
$$

This implies

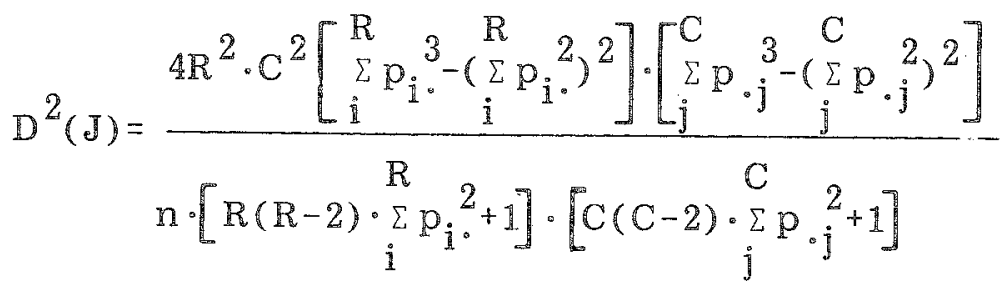

Assumption $p_{i}=1 / R$. What happens to $D^{2}(J)$ when the numerator of Equation 50 equals 0 ? It may be verified that this happens if and only if all $p_{i}$. are equal or all $p_{. j}$ are equal, i.e., when one marginal has equal proportions for the categories. Let it be assumed that $p_{i} .=1 / R, i=1,2, \ldots, R$. This implies

$$
\begin{aligned}
& \sum_{i}^{R} p_{i \circ}^{2}=\frac{1}{R} \\
& S_{R}=\sqrt{R-1}
\end{aligned}
$$

From this follows

$$
\mathbb{E}(J)=\frac{\sqrt{R-1} \cdot C \cdot\left(1-\sum_{j} p_{0 j}^{2}\right)}{(n-1) \cdot \sqrt{C(C-2) \sum_{j}^{C} p_{j j}^{2}+1}}
$$

From Equation 39 follows

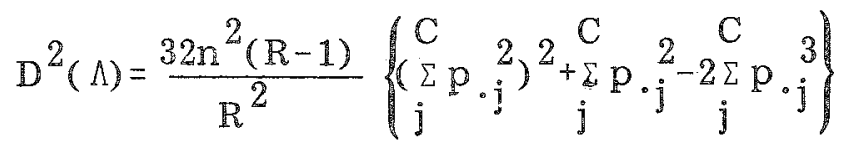

which implies

$$
D^{2}(J)=\frac{2 C^{2}}{C(C-2) \sum_{j} p_{\circ j}^{2}+1}\left[\left(\sum_{j} p_{\circ j}^{2}\right)^{2}+\sum p_{j}^{2}-2 \sum p_{j}^{2}{ }_{j}^{3}\right] \frac{1}{n^{2}}
$$

Assumption $p_{i}=1 / \mathbb{R}, p_{\cdot j}=1 / C$. Let finally both marginals consist of equal proportions. This means that $p_{\cdot j}=1 / C, j=1,2, \ldots, C$ together with assumption $p_{i} .=1 / R$. Then 
$E(J)=\frac{\sqrt{R-1} \cdot \sqrt{C-1}}{(n-1)}$

and

$$
\mathbb{D}^{2}(\mathbb{J})=\frac{2}{n^{2}}
$$

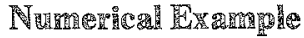

Suppose two judges have assigned 500 units, considered as randomly sampled from an infinitely large population, into categories. Each judge chose to use three categories, but there was no predefined relationship between the two sets of categories. The problem of estimating I will be considered. The result is summarized in Table 2.

\begin{tabular}{|c|c|c|c|c|c|}
\hline \multirow{2}{*}{\multicolumn{2}{|c|}{ Judge A }} & \multicolumn{4}{|c|}{ Judge B } \\
\hline & & $\begin{array}{l}\text { Cate- } \\
\text { gory } 1\end{array}$ & $\begin{array}{l}\text { Cate- } \\
\text { gory } 2\end{array}$ & $\begin{array}{l}\text { Cate- } \\
\text { gory } 3\end{array}$ & Sum \\
\hline Category & 1 & 10 & 60 & 30 & 100 \\
\hline Caregory & 2 & 70 & 120 & 10 & 200 \\
\hline Category & 3 & 20 & 20 & 160 & 200 \\
\hline Sum & & 100 & 200 & 200 & 500 \\
\hline
\end{tabular}

Assume a multinomial distribution, so that Equation 28 gives an asymptotic standard deviation of .03042 and the corresponding approximate $95 \%$ confidence interval of $I$ will be

$J \pm 1.96 \hat{\sigma}_{J}=.315 \pm .060=(.255, .375)$.

If, instead, a fixed marginal assumption is made, Equation 50 gives an asymptotic standard error of .00248. ${ }^{1}$ This assumption means that the judges had decided beforehand how many to assign to each category. The confidence interval here will thus be

$.315 \pm .005=(.310, .320)$.

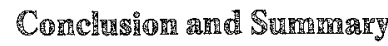

Hubert's $\Gamma$ is an interesting coefficient for measuring response agreement for nominal scales. Some of its properties may be considered as less suitable. An easy way of modifying it (creating $\Gamma^{*}$ ) eliminates a few of these properties. Another approach is to use the $J$-index, which is rather similar to $\Gamma$ but without these characteristics. $\mathbb{J}$ will thus always equal zero when all cell frequencies of the contingency table are equal.

\footnotetext{
${ }^{1}$ The computations of this section may be made by the CONTIN.program (Vegelius, 1978b).
} 


\section{References}

Brennan, R. L., \& Light, R. J. Measuring agreement when two observers classify people into categories not defined in advance. British Journal of Mathematical and Statistical Psychology, 1974, 27, 154-163

Cohen J. A coefficient of agreement for nominal scales. Educational and Psychological Measurement, 1960, 20, 37-46.

Cohen, J. Weighted kappa: Nominal scale agreement with provision for scaled disagreement or partial credit. Psychological Bulletin, 1968, 70, 213-220.

Cramér, H. Mathematical methods of statistics. Uppsala: Almqvist \& Wiksell, 1945.

Holley, J. W. \& Guilford, J. P. A note on the G index of agreement. Educational and Psychological Measurement, 1964, 24, 749-753.

Hubert, L. Nominal scale response agreement as a generalized correlation. British Journal of Mathematical and Statistical Psychology, 1977, 30, 98-103.

Janson, S., \& Vegelius, J. On the applicability of truncated component analysis based on correlation coefficients for nominal scales. Applied Psychological Measurement, 1978,2, 135-145.

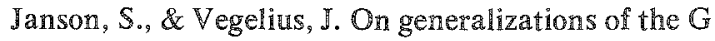
index and the phi coefficient to nominal scales. Multivariate Behavioral Research, 1979, 14 , 255-269.

Kendall, M. G. Rank correlation methods. London: Griffin, 1970 .

Rao, C. R. Linear statistical inference and its applications. New York: Willey, 1965.

Vegelius, J. On the utility of the $\mathbb{E}$-correlation coefincient concept in psychological research. $\mathbb{E} d u c a-$ tional and Psychological Measurement, 1978, 38, 605-611. (a)

Vegelius, J. CONTTN, a FORTRAN IV program for nominal scale correlation coefficients. Educational and Psychological Measurement, 1978, 38, $841-844$.

\section{Authorr's Addiress}

Send requests for reprints, further information, or copies of the CONTIN program to Jan Vegelius, Department of Statistics, Box 513, S-751 20 Uppsala, Sweden. 